\title{
Distale Radiusfraktur: Versorgungsstrategien beim älteren Menschen
}

\author{
Carsten Surke, Michael J. Raschke, Martin Langer
}

\section{Zusammenfassung}

Während beim jüngeren Patienten v.a. die Frakturmorphologie eine Herausforderung für den Operateur darstellt, müssen beim älteren Patienten weitere Aspekte mit in die Therapieplanung einfließen. Nebenerkrankungen und das soziale Umfeld müssen bei der Abwägung der optimalen Therapie ebenso bedacht werden wie die verminderte Knochenqualität. Steigende Ansprüche an die Funktion und Belastbarkeit aufgrund eines höheren Aktivitätsniveaus auch im Alter bilden dabei einen deutlichen Kontrast zur Hilflosigkeit des an Demenz erkrankten Patienten, der einer Versorgung nicht mit der notwendigen Compliance begegnen kann. Nur durch eine sorgfältige präoperative Abklärung sämtlicher Einflussfaktoren ist eine patientengerechte, individuelle Versorgung möglich.

\section{Einleitung}

Mit zunehmendem Alter steigt - insbesondere bei Frauen - die Wahrscheinlichkeit für das Vorliegen einer verminderten Knochenqualität und -stabilität durch die Osteoporose. Der Knochenumund -abbau kann am distalen Radius gut dargestellt werden. Durch Ausdünnung und Rarefizierung der Trabekel wird die Spongiosastruktur beim älteren Menschen erheblich minimiert (Abb. 1) [6].

Die im Alter vermehrte Inzidenz kardiovaskulärer Erkrankungen mit begleiten-

OP-JOURNAL 2012; 28: 256-260

(c) Georg Thieme Verlag KG Stuttgart · New York DOI http://dx.doi.org/10.1055/s-0032-1327997

\section{Distal Radial Fracture - Treatment Strategies for Elderly Patients}

The distal radial fracture is the most common fracture in adults. Different procedures exist ranging from conservative splinting to plate osteosynthesis. Whilst in younger patients the main challenge is posed by the fracture morphology itself, further aspects have to be taken into account in elderly patients. Secondary diseases and the social environment have to be considered as well as the reduced bone quality due to osteoporosis. On one side the increasing level of activity in the elderly leads to higher expectations in terms of function, on the other side a large proportion of elderly people suffers from dementia and is to some extent unable to comply with therapeutic necessities. Only with a profound knowledge of the patient's situation will the surgeon be able to choose an adequate path of treatment. Consideration of all these aspects is gaining additional importance in an ageing society. der orthostatischer Dysregulation und daraus folgenden Schwindelattacken erhöht gerade beim älteren Menschen die Sturzgefahr deutlich. Das Parkinson-Syndrom oder Polyneuropathien, z.B. als Folge eines langjährigen Diabetes mellitus, führen zu ausgeprägten Gangunsicherheiten. Die Einnahme neurotroper Medikamente (z.B. Antidepressiva, Schlafmittel) verstärkt diese Effekte weiterhin.

Die altersbedingt schwächere Muskulatur, die Verringerung der Reaktionsfähigkeit und der Reflexe sowie die durch degenerative Vorgänge verringerte Gelenkbeweglichkeit führen dazu, dass der Sturz häufig nicht mehr hinreichend und rechtzeitig abgefangen werden kann. Der osteoporotisch veränderte
Knochen hat weder die notwendige Festigkeit noch die Elastizität, den einwirkenden Kräften standzuhalten, und es kommt wesentlich leichter zur Fraktur [1].

\section{Hauptteil}

Generelles

Bei der Versorgung von distalen Radiusfrakturen des älteren Menschen müssen verschiedene Punkte in die Therapieplanung einfließen.

Neben der genauen Darstellung der Frakturmorphologie ist die Kenntnis des sozialen Umfelds genauso wichtig wie das Wissen um Komorbiditäten, die den Heilungsverlauf negativ beeinflussen können.

Im Rahmen der Behandlungsplanung muss der Chirurg entscheiden, ob der Patient in der Lage ist, ein u.U. restriktives Mobilisationsschema zu befolgen. Die Therapie wird unter Zusammenschau aller Aspekte unter Berücksichtigung des Alters und des Allgemeinzustands individuell für jeden Patienten einzeln festgelegt. Grundlage für eine adäquate Behandlung älterer Patienten ist eine interdisziplinäre Zusammenarbeit von Chirurg, Anästhesist und Geriater. Gerade in den letzten Jahren hat sich das Aktivitätsniveau der älteren Patienten und somit auch der funktionelle Anspruch an die Hand verändert. Diesen gestiegenen Ansprüchen bei einem immer größer werdenden Anteil in der älteren Bevölkerung sollte bei der Therapieplanung Rechnung getragen werden. Bei der Fraktur des distalen Radius beim alten Menschen kommt es zu einer irreversiblen Stauchung der schon osteoporotisch ausgedünnten Spongiosa, die im Rahmen der Reposition nach „Entstauchung“ der Fraktur als knöcherner Defekt deutlich wird. 


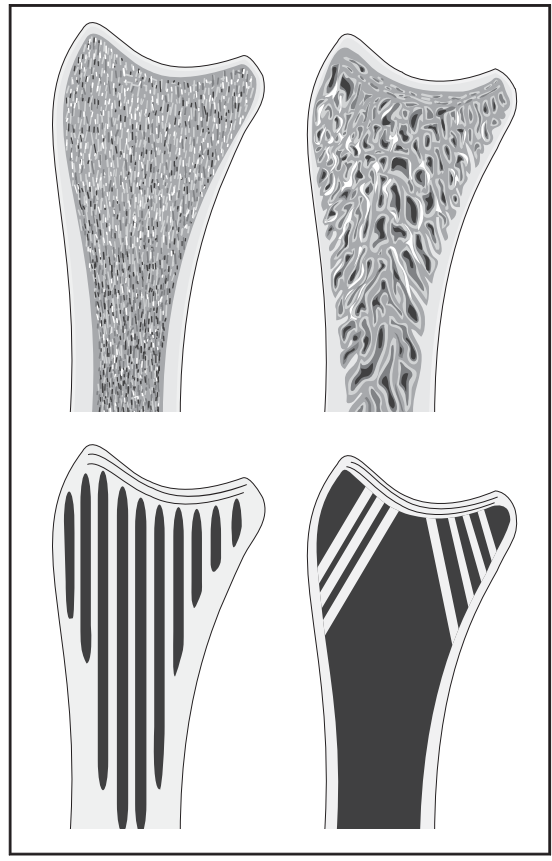

Abb. 1 Darstellung der trabekulären Struktur des distalen Radius im Sagittalschnitt beim Jugendlichen (links) im Vergleich zum älteren Patienten (rechts).

Daher gilt im höheren Alter besonders der Ausspruch: „Eine Fraktur, die reponiert werden muss, sollte auch operiert werden."

Die Altersgrenze für eine „stabile“ Reposition liegt etwa bei 50 Jahren. Eingestauchte Radiusfrakturen jenseits dieser Altersgrenze sintern zu einem großen Anteil wieder auf die ursprüngliche frakturbedingte Fehlstellung zurück [5]. Diagnostisch sollte die Indikation zur Dünnschicht-CT-Untersuchung des Handgelenks bei Verdacht auf intraartikuläre Frakturen zur besseren Planung der Versorgung großzügig gestellt werden.

\section{Therapie}

\section{Zeitfenster}

Wie bei nahezu allen frischen Verletzungen empfiehlt sich besonders beim älteren Patienten eine zügige Versorgung frischer distaler Radiusfrakturen. Bei Patienten, die unter dauerhafter Marcumartherapie stehen, sollte die Antikoagulation auf Heparin umgestellt werden. Je nach Operationsdringlichkeit kann der INR nach Rücksprache mit den Gerinnungsphysiologen durch Gerinnungsprodukte (z.B. PPSB) akut oder durch Vitamin-K-Substitution langsam angehoben werden. Die besten Eigenschaften für eine operative Therapie

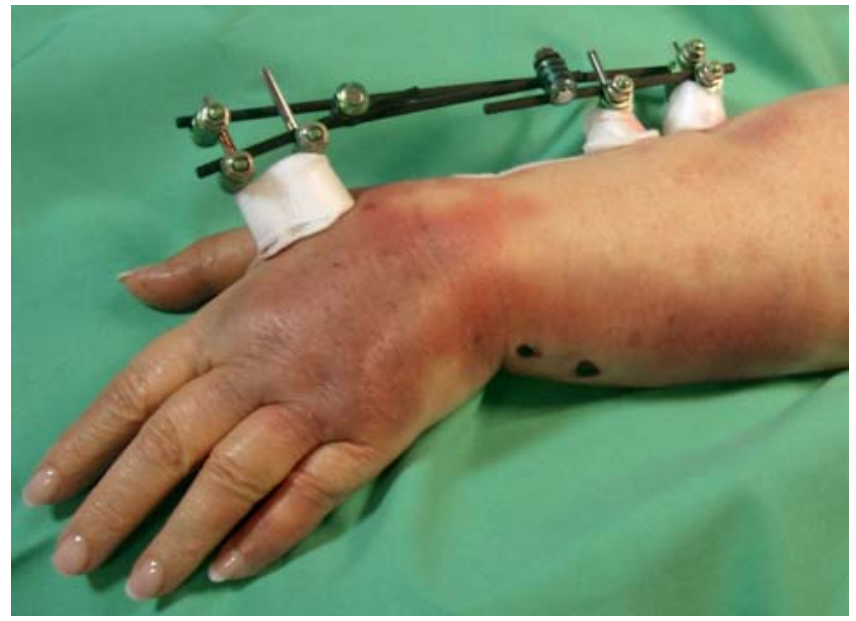

Abb. 2 Patientin mit angelegtem handgelenksübergreifendem Fixateur externe bei distaler Radiusfraktur.

bietet das Gewebe innerhalb der ersten 6 Stunden [6]. Später wird die Präparation durch die Gewebsschwellung und Verhärtung sowie durch in Organisation befindliche Hämatome deutlich schwieriger. Zudem wird nach etwa 1 Woche die Knochenqualität noch weiter durch ein „Aufweichen“ des im Hämatom liegenden Knochens schlechter, sodass die Schrauben fast ohne Bohrer eingedreht werden können. Ist eine zügige operative Versorgung nicht möglich, sollte zunächst die Ruhigstellung in einer Schiene erfolgen. Bei stark dislozierter Fraktur sollte diese unter adäquater Analgesie zunächst im Mädchenfänger ausgehangen, reponiert und anschließend in einer Unterarmschiene ruhig gestellt werden. Bei hochgradig instabilen Frakturen, die auch in einer Unterarmschiene nicht hinreichend stabil gehalten werden können, bietet sich überbrückend die Anlage eines handgelenksübergreifenden Fixateur externe (Abb.2) an. Im Anschluss sollte die Zeit zur Durchführung intensiver abschwellender Maßnahmen - wie Lymphdrainage und AV-Kompression genutzt werden. Die definitive operative Versorgung sollte dann innerhalb von 7 Tagen erfolgen.

\section{Konservative Therapie}

Die konservative Therapie sollte $v$.a. bei gering oder nicht dislozierten distalen Radiusfrakturen erwogen werden.

Die Reposition muss unter adäquater Analgesie erfolgen. Hierzu bieten sich die Bruchspaltanästhesie, die intravenöse Regionalanästhesie nach Bier, die Plexusanästhesie oder die Allgemeinnarkose an.

\section{Operative Therapie}

Jede stärker dislozierte oder instabile distale Radiusfraktur sollte operativ versorgt werden. Die Entscheidung, ab welchem Grad der Dislokation die Operation durchgeführt werden soll, trifft letztendlich der Chirurg, wobei die Kenntnis des sozialen Umfelds und der Komorbiditäten des Patienten in die Therapieplanung mit einfließen müssen. Zur Versorgung der distalen Radiusfraktur stehen verschiedene OP-Verfahren zur Verfügung.

\section{Anästhesie}

Die operative Versorgung distaler Radiusfrakturen erfolgt in Allgemein- oder Plexusanästhesie, wobei Letzterer v.a. beim älteren Menschen aufgrund ihrer geringen Komplikationsrate und der geringen Invasivität Vorzug gegeben werden sollte.

Der Eingriff erfolgt in Rückenlage, während der zu operierende Arm auf einen Handtisch in $90^{\circ}$-Abduktion ausgelagert ist. Durch diese Lagerung kann sowohl die palmare als auch die dorsale Seite des Radius adressiert werden.

\section{Kirschner-Draht-Osteosynthese}

Diverse Techniken der Kirschner-DrahtOsteosynthese (wie z.B. nach Willenegger) ermöglichen eine minimalinvasive Retention der distalen Radiusfraktur. Der Reposition einzelner (gerade intraartikulärer) Fragmente sind bei diesem Verfahren jedoch Grenzen gesetzt. Besonders beim osteoporotischen Knochen besteht die Gefahr der Lockerung der K-Drähte mit sekundärem Korrekturverlust. Bei Anwendung dieses Verfahrens ist präoperativ streng zu prüfen, ob der Patient in der Lage ist, eine lang 


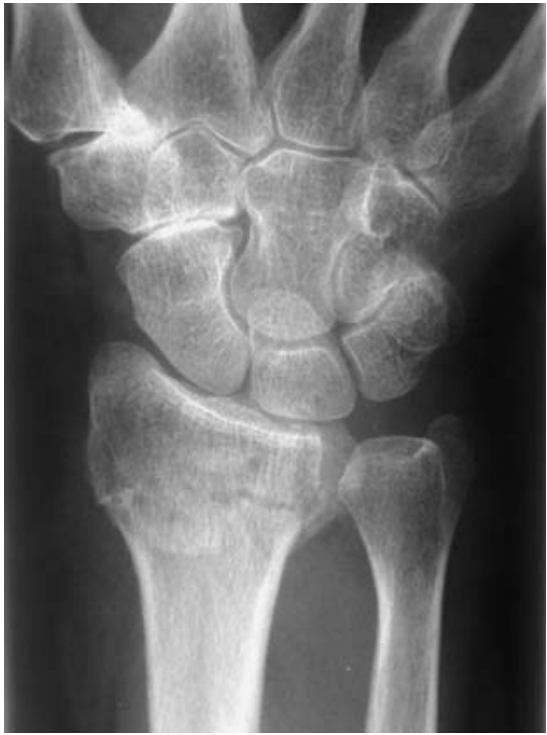

Abb. 3 Distale Radiusfraktur AO 23 A3 im d.p. Strahlengang.

andauernde Gipsruhigstellung zu tolerieren (Cave: Gefahr der Reflexdystrophie!).

\section{Fixateur externe}

Neben der Notfallstabilisierung eignet sich der handgelenksübergreifende Fixateur externe in einigen Fällen auch bei alten Menschen zur Ausbehandlung komplexer Frakturen. Hierbei werden je 2 Schanz-Pins im Radiusschaft und im Schaft des 2. Mittelhandknochens verankert und nach geschlossener Reposition der Fixateurbody montiert. Zur zusätzlichen Stabilisierung kann die Fraktur mit perkutanen K-Drähten stabilisiert werden. Auch hier besteht das Problem der Pinlockerung im osteoporo-

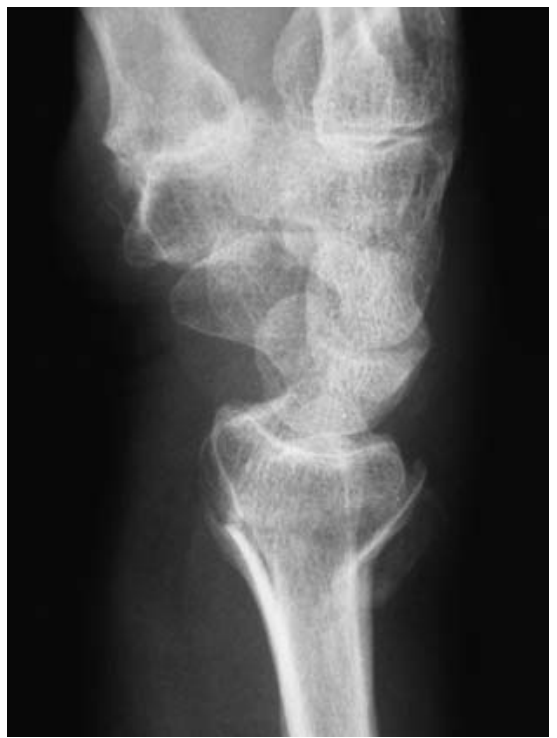

Abb. 4 Distale Radiusfraktur AO 23 A3 im lateralen Strahlengang.

tischen Knochen. Der Fixateur bleibt bei diesem Verfahren bis zur Frakturheilung in situ. Eine frühfunktionelle Nachbehandlung ist unmöglich. Gerade dem an Demenz erkrankten älteren Patienten ist häufig eine langzeitige Behandlung im Fixateur externe nicht zuzumuten, da hier eine hohe Gefahr der akzidentiellen Selbstverletzung besteht. Auch die im postoperativen Verlauf durchzuführende Pflege der Pineintrittstellen ist besonders für den älteren Patienten schwierig.

\section{Plattenosteosynthese}

Die meisten distalen Radiusfrakturen können mittels der palmaren winkelstabilen Plattenosteosynthese anatomisch rekonstruiert und stabil versorgt werden. Auch erlaubt diese Art der Versorgung bei fehlenden Begleitverletzungen eine frühfunktionelle Nachbehandlung. Die Verbesserung des Implantatdesigns, z.B. die polyaxiale Einbringung der Schrauben, hat dazu geführt, dass auch intraartikuläre Frakturen mit dorsaler Trümmerzone allein durch den palmaren Zugang stabil versorgt werden können. Exemplarisch ist diese Versorgung anhand eines Fallbeispiels einer 60 -jährigen Patientin in den Abb.3-11 dargestellt. Wir wählen zur Versorgung den radiopalmaren Zugang, wobei die Inzision radial der FCR-Sehne gesetzt wird. Unter Schonung der Sehnenscheide der FCR-Sehne erfolgt die Präparation in die Tiefe. Bei der weiteren Präparation werden die Beugesehnen zusammen mit dem von der FCR-Sehne geschützten N. medianus nach ulnar gehalten. Es erfolgt die Darstellung des M. pronator quadratus und die Abtrennung desselben von seinem sehnigen Ansatz am Radius bzw. an der Sehne des M. brachioradialis. Der M. pronator quadratus kann nun vom Radius abgeschoben und somit die Fraktur dargestellt werden. Die Reposition erfolgt unter Sicht, wobei dorsale Fragmente zumeist unter Nutzung der Ligamentotaxis eingestellt werden können.

Beim Aufbringen der winkelstabilen Osteosyntheseplatte muss darauf geachtet werden, diese proximal der palmaren Radiuskante (Watershed-Linie) zu platzieren. Ein Hinausragen der Platte über diese Linie hinaus kann zu Irritationen und nicht selten später zur Ruptur der Sehne des M. flexor pollicis longus oder des Flexor digitorum profundus des Zeigefingers führen.

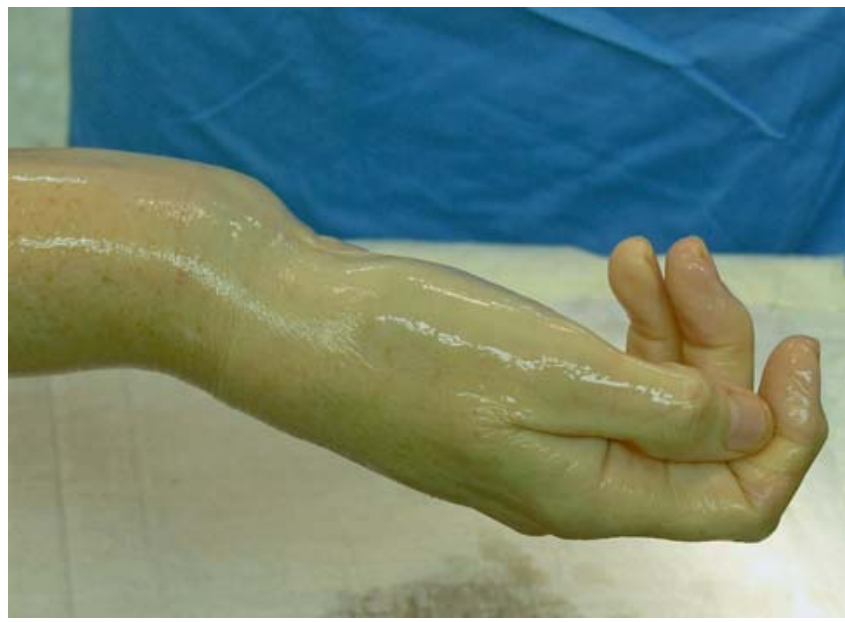

Abb.5 Klinischer Aspekt einer distalen Radiusfraktur mit typischer Bajonettstellung des Handgelenks.

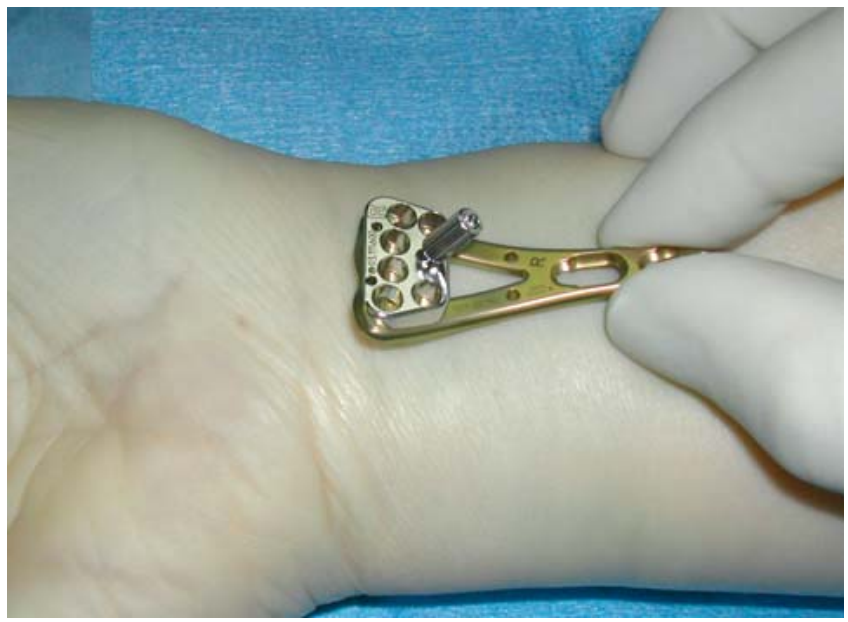

Abb. 6 Winkelstabile 2-Säulen-Platte (Fa. Synthes). 

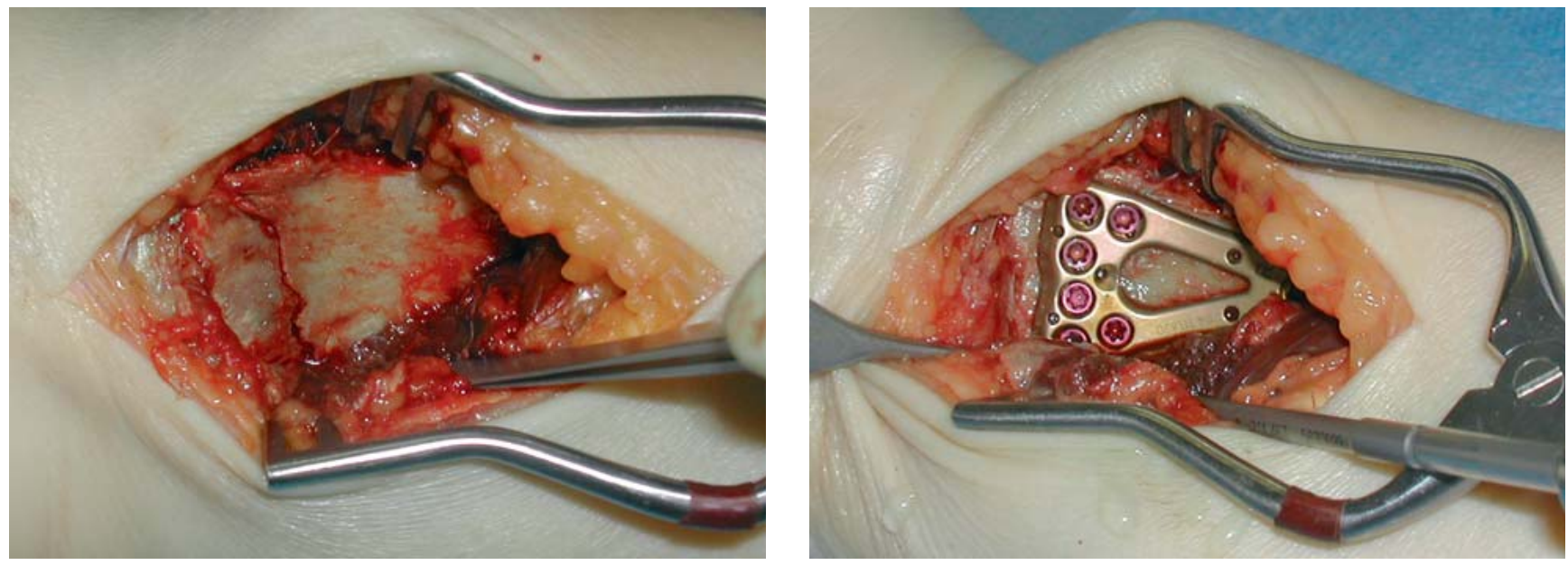

Abb. 7 Blick auf die Fraktur von palmar vor Reposition.

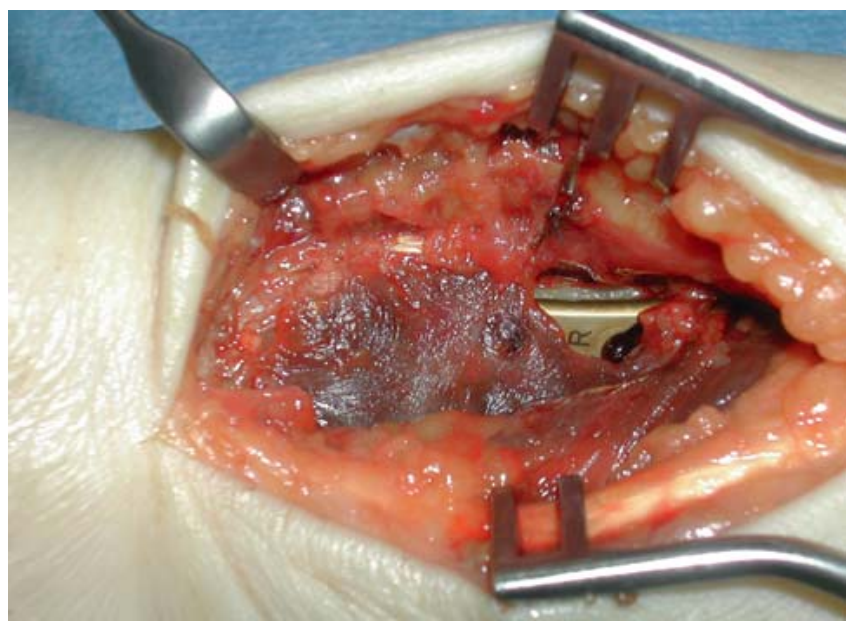

Abb. 9 Abdecken der Osteosyntheseplatte durch Refixation des M. pronator quadratus.
Die Schraubenlöcher am Plattenschaft können meist mit nicht winkelstabilen Schrauben besetzt werden, die distalen Plattenlöcher sollten zur besseren Abstützung der Gelenkfläche und Vermeidung eines Korrekturverlusts mit winkelstabilen Schrauben besetzt werden. Hierbei muss die Schraubenlänge so gewählt werden, dass die Schraubenspitzen nicht die dorsale Kortikalis überragen, da hierdurch die Strecksehnen irritiert werden können.

Nicht selten werden durch zu lange Schrauben die Strecksehnen derart verletzt, dass es im Verlauf zu sekundären Rupturen kommt. Aus diesem Grund empfehlen wir beim Setzen der distalen Osteosyntheseschrauben auf eine Perforation der dorsalen Radiuskortikalis zu verzichten.

Zur Kontrolle empfehlen wir eine tangentiale Röntgenaufnahme des dorsalen Handgelenks, die 2005 in Jena entwickelt wurde (Abb. 12 und 13). Abschließend kann die Osteosyntheseplatte durch Re- fixation des M. pronator quadratus an seinem Ansatz zumeist sicher abgedeckt werden.

Der dorsale Zugang zum Handgelenk findet besonders bei Frakturen mit dorsaler Pathologie wie z.B. der Barton-Fraktur Anwendung. Außerdem kann über den dorsalen Zugang eine palmar nicht hinreichend stabilisierte Fraktur zusätzlich abgestützt werden. Ein weiterer Vorteil dieses Zugangs ist die gute Einsicht in die radiokarpale Gelenkfläche. Trotz deutlicher Verbesserung des Implantatdesigns mit Einführung schlanker, anatomisch präformierter, winkelstabiler Implantate sollte dieser Zugang aufgrund seines hohen Grades an Weichteiltraumatisierung mit der Gefahr der Strecksehnenirritation und -verklebung möglichst vermieden werden.

Am Ende der Operation sollte eine dynamische Durchleuchtung erfolgen, um karpale Instabilitäten aufzudecken oder auszuschließen.

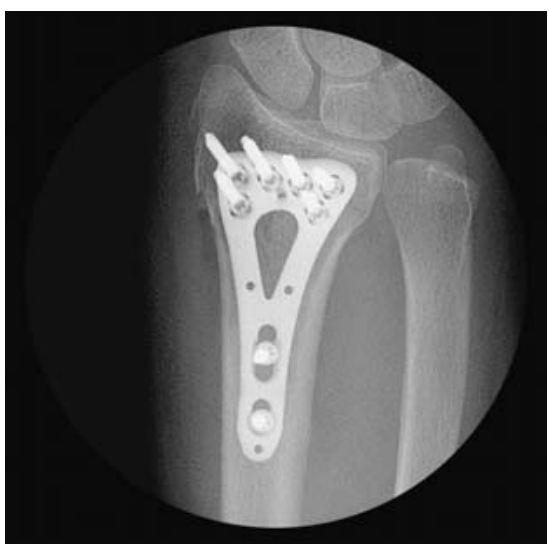

Abb. 10 Postoperative Röntgenkontrolle im d. p. Strahlengang.

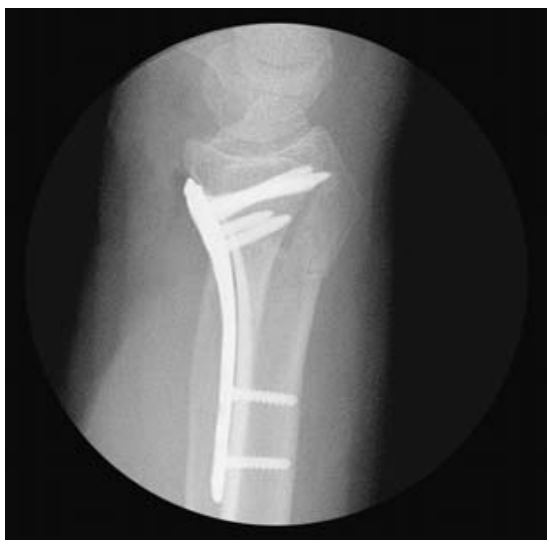

Abb. 11 Postoperative Röntgenkontrolle im lateralen Strahlengang.

Hier ist v.a. die SL-Band-Ruptur zu nennen. Aber auch Instabilitäten des distalen Radioulnargelenks können bei der Durchleuchtung nach der Stabilisierung des Radius aufgedeckt werden. 


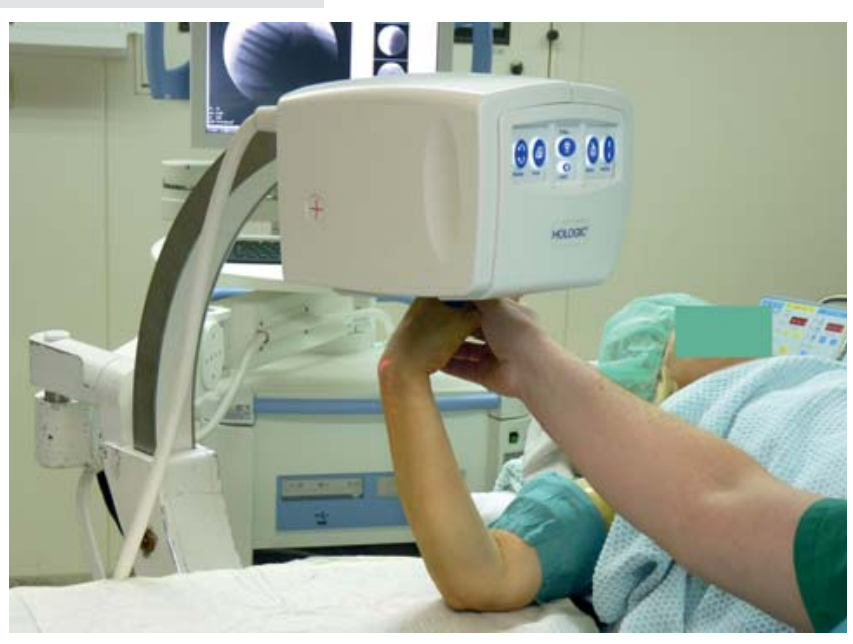

\section{Nachbehandlung}

Wichtigstes Ziel einer Versorgung muss die möglichst frühzeitige funktionelle Nachbehandlung sein. Bei stabiler palmarer Plattenosteosynthese sollte mit einer frühfunktionellen Nachbehandlung zügig begonnen werden. Je nach Begleitverletzung, Compliance oder Knochenqualität kann eine zusätzliche Unterarmschiene oder Orthese für einige Tage bis Wochen notwendig werden.

\section{Schlussteil}

Verschiedene Aspekte müssen bei der Wahl des Therapieregimes bedacht werden. Die osteoporotisch bedingt schlechte Knochenqualität muss genauso in die Therapieplanung einfließen wie Komorbiditäten und das soziale Umfeld des Patienten.

Auch weiterhin wird die ideale Versorgungsstrategie der distalen Radiusfraktur beim älteren Menschen diskutiert. Operative wie konservative Verfahren zeigen ähnliche Langzeitergebnisse [7]. Während bei der Versorgung komplexer intraartikulärer Trümmerfrakturen Kapoor et al. im langfristigen Verlauf die Behandlung mittels Fixateur externe favorisieren [4], scheint bei metaphysären
Abb. 12 Technik der Tangentialaufnahme der dorsalen Radiuskortikalis. im langfristigen Verlauf weder die konservative Behandlung im Gips, noch die Osteosynthese mittels Fixateur externe, noch die Plattenosteosynthese klare Vorteile aufzuweisen [1].

Im kurzfristigen Verlauf (3 bis 6 Monate) erlaubt allerdings die winkelstabile palmare Plattenosteosynthese der dislozierten distalen Radiusfraktur eine frühere Mobilisierung aufgrund der höheren Primärstabilität [1]. Gerade vor diesem Hintergrund sollte unserer Meinung nach der winkelstabilen Plattenosteosynthese beim älteren Menschen der Vorzug gegeben werden.

Die Plattenosteosynthese erlaubt eine deutlich höhere Selbstständigkeit des alten Menschen (ADL) und verhindert wirkungsvoll die Ausbildung eines CRPS.

Die Therapie muss individuell an die Patientensituation angepasst werden.

\section{Literatur}

${ }^{1}$ Brug E, Joosten U, Püllen $M$. Brüche am distalen Unterarm: Welche Therapie ist wann indiziert? Orthopäde 2000; 29: 318-326

2 Egol KA, Walsh M, Romo-Cardoso S et al. Distal radial fractures in the elderly: operative compared with nonoperative treatment. J Bone Joint Surg [Am] 2010; 92: 1851-1857

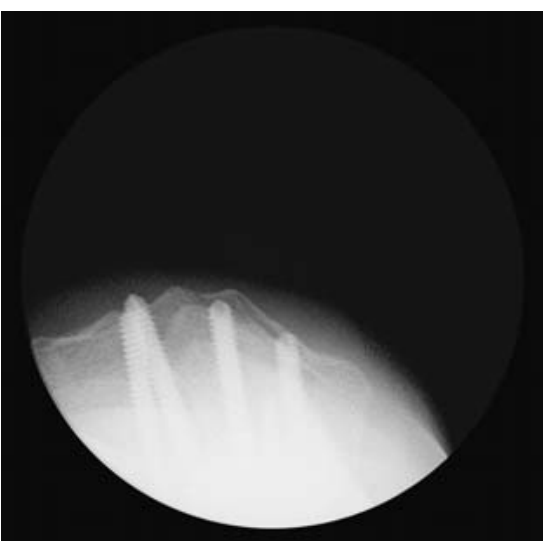

Abb. 13 Darstellung der dorsalen Radiuskortikalis im tangentialen Röntgenbild. Gut zu erkennen ist die Perforation der Kortikalis im 3. Strecksehnenfach durch die 2. Schraube von links.

${ }^{3}$ Jupiter JB. Fractures of the distal end of the radius. J Bone Joint Surg [Am] 1991; 73: 461469

${ }^{4}$ Kapoor H, Argawal A, Dhaon BK. Displaced intra-articular fractures of the distal radius: a comparative evaluation of results following closed reduction, external fixation and open reduction with internal fixation. Injury 2000: 31: 75-79

${ }^{5}$ Nesbitt KS, Failla JM, Clifford L. Assessment of instability factors in adult distal radius fractures. J Hand Surg [Am] 2004; 29: 1128-1138

${ }^{6}$ Raschke MJ, Stange R. Alterstraumatologie. München: Urban \& Fischer; 2008

7 Wie DH, Raizman NM, Bottino CJ et al. Unstable distal radial fractures treated with external fixation, a radial column plate, or a volar plate. A prospective randomized trial. J Bone Joint Surg [Am] 2009; 91: 1568-1577

\section{Dr. med. Carsten Surke}

Facharzt

Prof. Dr. med. Michael J. Raschke

Direktor der Klinik

Priv.-Doz. Martin Langer

Sektionsleiter Handchirurgie

Klinik für Unfall-, Hand- und

Wiederherstellungschirurgie

Albert-Schweitzer-Campus 1,

Gebäude W1

Anfahrtsadresse: Waldeyerstraße 1 48149 Münster

Carsten.Surke@ukmuenster.de 